\title{
When Memory Does Not Fail: Familiarity-Based Recognition in Mild Cognitive Impairment and Alzheimer's Disease
}

\author{
Carmen E. Westerberg, Ken A. Paller, \\ Sandra Weintraub, and M.-Marsel Mesulam \\ Northwestern University
}

Andrew R. Mayes

University of Manchester

\author{
Juliet S. Holdstock \\ University of Liverpool
}

\author{
Paul J. Reber
}

Northwestern University

\begin{abstract}
Recognition can be guided by familiarity, a restricted form of retrieval devoid of contextual recall, or by recollection, which occurs when retrieval is sufficient to support the full experience of remembering an episode. Recollection and familiarity were disentangled by testing recognition memory using silhouette object drawings, high target-foil resemblance, and both yes-no and forced-choice procedures. Theoretically, forced-choice recognition could be mediated by familiarity alone. Alzheimer's disease and its preclinical stage, mild cognitive impairment (MCI), were associated with memory impairments that were greater on the yes-no test. Remarkably, forced-choice recognition was unequivocally normal in patients with MCI compared with age-matched controls. Neuropathology in hippocampus and entorhinal cortex, known to be present in MCI, presumably disrupted recollection while leaving familiarity-based recognition intact.
\end{abstract}

Keywords: hippocampus, entorhinal cortex, forced-choice recognition, recollection, familiarity

\begin{abstract}
Alzheimer's disease (AD) is associated with deficits in several cognitive domains, including episodic memory, and these deficits increase in severity with disease progression (e.g., Morris, 1996). A prodromal stage of $\mathrm{AD}$ that typically includes relatively isolated memory impairments has been designated as mild cognitive impairment (MCI; Petersen et al., 2001). Not all patients with MCI develop $\mathrm{AD}$, but all $\mathrm{AD}$ patients pass through a prodromal MCIlike stage. MCI is often distinguished from early dementia on the basis of preserved daily living activities. Although the Petersen criteria emphasize the importance of memory loss relative to other deficits, recent accounts have pointed out that MCI can occur with other cognitive impairments in addition to memory or in a single cognitive domain other than memory, with daily living activities relatively preserved (Luis, Loewenstein, Acevedo, Barker, \& Duara, 2003; Winblad et al., 2004). Despite the high incidence of memory deficits in MCI, the precise nature of these impairments remains to be elucidated.
\end{abstract}

Carmen E. Westerberg, Ken A. Paller, and Paul J. Reber, Department of Psychology and Institute for Neuroscience, Northwestern University; Sandra Weintraub and M.-Marsel Mesulam, Institute for Neuroscience and Cognitive Neurology and Alzheimer's Disease Center, Northwestern University; Juliet S. Holdstock, School of Psychology, University of Liverpool, Liverpool United Kingdom; Andrew R. Mayes, School of Psychological Sciences, University of Manchester, Manchester, United Kingdom.

This research was supported by National Institutes of Health Grants AG20506, NS34639, and MH58748 and by Northwestern University Cognitive Neurology and Alzheimer's Disease Center Grant P30 AG13854 from the National Institute on Aging. We thank Ken Norman and Andy Yonelinas for helpful discussions regarding this article and Anjali Raja for her assistance with data collection.

Correspondence concerning this article should be addressed to Carmen E. Westerberg, Department of Psychology, Northwestern University, 2029 Sheridan Road, Evanston, IL 60208. E-mail: c-westerberg@ northwestern.edu
Memory is an exceedingly complex aspect of cognition based on multiple interrelated processes that vary in their dependence on neuroanatomical substrates. Clinical conditions therefore frequently display dissociations among the types of memory that are impaired versus those that are preserved. In one study, patients with AD showed some ability to discriminate previously presented words from novel words but performed at a chance level when asked to identify the color of each recognized word (Tendolkar et al., 1999). Such dissociations may highlight systematic differences in the nature of information-processing dysfunction.

Memory dissociations in neurological patients have sometimes been described with reference to theories postulating that two distinct memory phenomena can contribute to the recognition of past experience, recollection and familiarity (Mandler, 1980; Yonelinas, 2002). Similarly, one can distinguish between item memory, which pertains to an object or stimulus at the focus of attention, and source memory, which pertains to contextual information (such as a spatial location or timing of a prior event, other features of the environment, a progression of immediately preceding events, and so on). Recollection entails retrieving information not presented as a recognition cue, especially the source information that defines the learning episode. Familiarity refers to item memory devoid of any recall of source information.

Recollection and familiarity may have a relationship of one-way redundancy. Familiarity may occur in the absence of recollection, as in the butcher-on-the-bus phenomenon, whereby an individual in an atypical setting is recognized but disambiguating contextual information is not retrieved (Mandler, 1980; Yovel \& Paller, 2004) - but perhaps recollection does not occur without familiarity (Tulving, 1989). Another possibility is that recollection and familiarity are independent, such that items may evoke recollection without necessarily evoking familiarity (Yonelinas, 1999). 
Computationally based claims about the kind of processing strategies that underlie recollection and familiarity have been put forward with reference to the Complementary Learning Systems model (McClelland, McNaughton, \& O'Reilly, 1995; Norman \& O'Reilly, 2003). According to this model, recollection is mediated by a system that assigns relatively distinct representations to individual stimuli. This processing strategy is beneficial for rapid learning without interference from other, similar stimuli because experience with a single stimulus strengthens the representation for that stimulus with little effect on the representations for other, similar stimuli. Furthermore, this strategy allows the system to mediate pattern completion, whereby partial information about an episode can cue the retrieval of missing information. Unlike recollection, which involves the retrieval of qualitative information about the study event associated with a recognition cue, familiarity is based on quantitative variations in the memory strength of the cue itself. According to the model, familiarity is mediated by a system that assigns overlapping representations to individual stimuli, and the amount of overlap varies as a function of the similarity between different stimuli. Therefore, the familiarity of an item that has not been encountered but is similar to other, previously encountered items may be quite high due to the high level of representational overlap between similar items. Over time, this is helpful for organizing categories based on similarity and for generalizing to novel stimuli. Memory for information that is common to several similar items is often referred to as gist memory (e.g., Reyna \& Brainerd, 1995; Schacter, Norman, \& Koutstaal, 1998). When several similar stimuli are encountered, familiarity is better suited to support memory for gist than recollection, but it is important to keep in mind that experience with a specific stimulus increases familiarity not only for gist information shared by similar stimuli but also for item-specific information uniquely associated with that stimulus.

In many situations, both recollection and familiarity can support effective recognition performance. However, in a yes-no recognition test with targets and foils that share a high level of perceptual overlap, the familiarity system cannot guide accurate performance if memory strength among different targets varies significantly. Yet familiarity can be effective in a forced-choice recognition test when each target is grouped with foils that are perceptual variants of that target. Familiarity can lead to a correct response in this forced-choice format because (a) direct comparisons of relative familiarity are facilitated by the simultaneous presentation of the target and its corresponding foils and (b) the variation in memory strength across different target items need not impact recognition decisions because all foils corresponding to a given target are evaluated simultaneously with that target. In sum, when memory is tested under these circumstances, familiarity may effectively support forced-choice recognition but fail to effectively support yes-no recognition. Recollection, on the other hand, can support both yes-no and forced-choice recognition, but this support wanes when correct responding depends on minute stimulus details that are not reliably brought back to mind when a study episode is recollected.

The failure of familiarity-based responding in yes-no recognition with high target-foil similarity can be conceptualized as follows. When target-foil similarity is high, target memory representations are not distinctive because they overlap with foil representations. It is reasonable to also assume that some targets may engender much higher familiarity signals than other targets. Foils corresponding to targets with high familiarity can thus produce familiarity signals that exceed those from targets with low familiarity. Accordingly, assigning a threshold above which test items are given a "yes" response is problematic. Many foils will exceed the familiarity threshold, and/or many targets will fail to reach this threshold, resulting in poor yes-no recognition performance.

In contrast, familiarity can still support accurate performance on forced-choice tests when targets are grouped with foils that are highly similar. The familiarity of each target and its corresponding foils may be very similar, but the familiarity of a specific target will be slightly yet reliably higher than the familiarity of its corresponding foils. In these forced-choice tests, the individual need only select the item with the highest familiarity from among a group of similar items.

Consistent with this idea, Bastin and Van der Linden (2003) tested yes-no and forced-choice recognition with targets and foils that were similar and estimated the contribution of recollection and familiarity to both kinds of recognition judgments using the remember-know-guess procedure (Gardiner, Java, \& RichardsonKlavehn, 1998). Familiarity contributed more to forced-choice than to yes-no recognition. On the other hand, using the remember-know procedure (Tulving, 1985), no differences in the relative contributions of recollection and familiarity were found between yes-no and forced-choice recognition using unrelated targets and foils (Khoe, Kroll, Yonelinas, Dobbins, \& Knight, 2000). These results suggest that the utility of familiarity is greater in forcedchoice recognition than in yes-no recognition only when targets and foils are similar.

Recent evidence from neuropsychological investigations indicates that distinct neural systems may support recollection and familiarity. In patients with damage limited to the hippocampus, recall was clearly impaired, but in some patients, item recognition was apparently intact or at least relatively intact (Aggleton \& Brown, 1999; Mayes, Holdstock, Isaac, Hunkin, \& Roberts, 2002; Vargha-Khadem et al., 1997). These findings have prompted speculation that a hippocampal contribution is necessary for normal performance when memory tasks require recollection, but is not necessary to the extent that familiarity is sufficient by itself.

To further assess the role of the hippocampus in memory processing, Holdstock and colleagues (2002) measured recall and recognition memory performance in a patient with focal pathology restricted to the hippocampus (Patient YR). Results indicated that memory was impaired in YR for various recall tests and for yes-no object recognition with similar targets and foils but not for forcedchoice object recognition when targets were grouped with corresponding foils. Similarly, Yonelinas and colleagues (2002) studied hypoxic patients (who had suffered brain damage thought to be limited to the hippocampus) and patients with more extensive medial temporal damage due to other insults. Hypoxic patients showed intact familiarity, whereas patients with more extensive medial temporal damage showed impaired familiarity, consonant with the view that the contribution of the hippocampus to familiarity is minimal. Additional evidence likewise indicates that patients with damage restricted to the hippocampus can show relatively preserved familiarity with impaired recollection (Aggleton et al., 2005; Baddeley, Vargha-Khadem, \& Mishkin, 2001; Bastin et al., 2004; Holdstock et al., 2002; Mayes et al., 2004). Studies that have assessed recognition in patients with extensive medial 
temporal damage have found that these patients generally exhibited recognition deficits in both recollection and familiarity (Giovanello \& Verfaellie, 2001; Knowlton \& Squire, 1995).

The findings across these neuropsychological studies are fairly consistent with the notion that hippocampal damage leads to impaired recollection, whereas damage that extends more broadly through the medial temporal lobe leads to deficits in both recollection and familiarity. However, it is noteworthy that Manns, Hopkins, Reed, Kitchener, and Squire (2003) obtained results in patients with relatively selective hippocampal damage suggesting that both recollection and familiarity were impaired. One popular hypothesis that nonetheless requires further empirical support is that familiarity depends on the integrity of structures located adjacent to the hippocampus, within the area that can be referred to as medial temporal cortex (MTC). The anterior portion of MTC includes entorhinal and perirhinal cortices, and the posterior portion of MTC includes parahippocampal cortex (Amaral, 1999).

Evidence from functional magnetic resonance imaging (fMRI) also indicates that the hippocampus may be more critical for recollection than for familiarity. Subsequent memory analyses have revealed that encoding that led to recollection recruited greater hippocampal activity than encoding that led to familiarity without recollection (Davachi, Mitchell, \& Wagner, 2003; Ranganath et al., 2004). During test, recognition based on recollection produced greater hippocampal activation compared with recognition based on familiarity (Eldridge, Knowlton, Furmanski, Bookheimer, \& Engel, 2000; Yonelinas, Hopfinger, Buonocore, Kroll, \& Baynes, 2001).

There is some evidence that MTC plays a role in familiarity. Perirhinal cortex, in particular, has been implicated in familiarity processing by some fMRI studies, although others have failed to find any familiarity-related medial temporal activity. Neural events during encoding tend to show greater perirhinal activity for items subsequently recognized with familiarity than for items subsequently forgotten (Davachi et al., 2003; Ranganath et al., 2004; but see Henson, Rugg, Shallice, \& Dolan, 1999). During retrieval, most studies have failed to find statistically significant perirhinal activity correlated with familiarity (Eldridge et al., 2000; Henson et al., 1999; Yonelinas et al., 2001). However, a meta-analysis of four fMRI experiments indicated that perirhinal cortex activity during retrieval was reliably decreased for familiar items compared with unfamiliar items, although these effects were missed in initial analyses for each experiment (Henson, Casino, Herron, Robb, \& Rugg, 2003).

Additional evidence from other domains also suggests that perirhinal cortex may be a critical structure for familiarity. Singleunit responses within this region decrease as familiarity of an item increases, conceivably comprising a code for item familiarity (Brown \& Xiang, 1998). Also, ablation studies with monkeys have demonstrated greater recognition deficits following conjoint perirhinal and parahippocampal lesions than following hippocampal or entorhinal lesions (Leonard, Amaral, Squire, \& Zola-Morgan, 1995; Zola-Morgan, Squire, Amaral, \& Suzuki, 1989; ZolaMorgan, Squire, \& Ramus, 1994), consistent with the possibility that perirhinal cortex plays an important role in familiarity. Yet some evidence suggests that multiple MTC regions may be crucial for familiarity (Yonelinas et al., 2002), and the possibility that other cortical regions contribute to familiarity (Henson et al., 1999) cannot be ruled out.
Neuropathological investigations of AD and MCI have indicated that the earliest signs of disease appear in the hippocampus and entorhinal cortex. The major neuropathological markers of AD are amyloid plaques and neurofibrillary tangles (NFTs) found in larger numbers than those observed in brains of nondemented individuals (Braak \& Braak, 1991). Recent evidence indicates that NFT density is also greater in MCI than in normal cognitive aging, although differences in the density of amyloid plaques were not observed (Guillozet, Weintraub, Mash, \& Mesulam, 2003). The distribution of NFTs is primarily limited to the hippocampus and entorhinal cortex in these cases early on and gradually becomes more widespread with disease progression (Delacourte et al., 1999; Mesulam, 1999). Furthermore, NFT density in the medial temporal lobe is strongly correlated with memory dysfunction (Guillozet et al., 2003). A recent study reported significantly higher tangle counts in the nucleus basalis of Meynert in individuals who were followed longitudinally and who, prior to death, had shown evidence of a change from cognitively normal to MCI compared with cognitively normal individuals who remained that way until death (Mesulam, Shaw, Mash, \& Weintraub, 2004). It was suggested that this early involvement of the cholinergic system could account for what is considered to be age-related cognitive change. Significant atrophy in hippocampus and entorhinal cortex in MCI and AD is also evident in structural MRI (e.g., Du et al., 2001; Killiany et al., 1993; Seab et al., 1988). Some findings have suggested that entorhinal atrophy exceeds that of hippocampus in MCI (Pennanen et al., 2004) and AD (Du et al., 2001; but see Du et al., 2004; Xu et al., 2000), and entorhinal atrophy has been implicated as a risk factor for the development of AD (Stoub et al., 2005). In addition, significant neuronal loss has been demonstrated in hippocampus and entorhinal cortex in mild AD (Gomez-Isla et al., 1996; West, Coleman, Flood, \& Troncoso, 1994) and in entorhinal cortex in MCI (Kordower et al., 2001).

The pathology of AD typically includes damage extending beyond medial temporal regions into several regions of neocortex (Braak \& Braak, 1991). Cortical hypometabolism (e.g., de Leon et al., 1983; Foster et al., 1984; Friedland et al., 1989; Grady et al., 1988; Ibáñez et al., 1998) and NFTs (Braak \& Braak, 1991; Delacourte et al., 1999) can also be found throughout temporal, parietal, and frontal cortex in individuals with AD.

Understanding the precise nature of memory impairments in individuals with $\mathrm{AD}$ and MCI can thus be quite informative. Advances in neurocognitive conceptualizations of familiarity, recollection, and recognition deficits may also have practical value for considering the memory loss these patients experience and for dealing with its impact on daily life.

In the present study, patients with $\mathrm{AD}$, patients with $\mathrm{MCI}$, and healthy older control participants were administered the yes-no and forced-choice recognition tests used by Holdstock and colleagues (2002), in which foils are highly similar to targets. It was predicted that medial temporal pathology in both patient groups would result in yes-no recognition impairments. Consistent with this prediction, recent evidence indicates that patients with $\mathrm{AD}$ are impaired at yes-no recognition for categorized color photographs (Budson et al., 2003) and perceptually similar novel objects (Budson, Desikan, Daffner, \& Schacter, 2001). If intact hippocampal and entorhinal tissue is critical for familiarity, then AD and MCI patients should also show impairments on the forced-choice test. However, if intact hippocampal and entorhinal tissue is not critical 
for familiarity, then $\mathrm{AD}$ and MCI patients might show preserved forced-choice recognition. If neural tissue damaged in $\mathrm{AD}$ but intact in MCI is critical for familiarity, then only AD patients should show forced-choice recognition impairments. Yes-no and forced-choice recognition tests were also administered to young adults with either a 3-min, 1-week, or 2-week retention delay to clarify how performance on the two tests declines when memory is weak due to normal decay over time, in the absence of any underlying memory disorder. Differences in the pattern of memory performance across the two tests between young healthy adults tested at long delays and MCI and AD patients should highlight the special nature of the memory disorders in MCI and AD.

\section{Method}

\section{Participants}

Participants were recruited from the Northwestern University Cognitive Neurology and Alzheimer's Disease Center. Exclusion criteria were history of central neurological disease, major psychiatric disorder or alcohol or substance abuse according to criteria from the Diagnostic and Statistical Manual of Mental Disorders (4th ed. [DSM-IV]; American Psychiatric Association, 1994), serious medical illness (thyroid disorder; renal, hepatic, cardiac, or pulmonary insufficiency; unstable diabetes; uncontrolled high blood pressure; cancer), and chronic use of psychoactive or hypnotic medications. Participants with medication-controlled hypertension or hypercholesterolemia were included.

The two patient groups included 8 individuals ( 5 women, 3 men) diagnosed with dementia according to $D S M-I V$ diagnostic criteria and probable AD based on research diagnostic criteria (McKhann et al., 1984) and 8 individuals ( 6 women, 2 men) diagnosed with MCI. All MCI participants were impaired on memory tests, and some had scores on the Boston Naming Test that were not considered normal for age, such that they fell into the category of amnestic-multiple domain MCI (Petersen et al., 2001; Petersen, 2004). Cutoff scores for abnormality on each neuropsychological test were two standard deviations below the mean score for the relevant age and education group (wherever available). This highly conservative approach resulted in seemingly low scores for some patients; for example, MCI Patient 7 in Table 1 had a Constructions score of 8 that was considered normal, but a score of 8 was considered impaired for two younger AD patients.

For each patient, an informant provided objective information in an interview about daily living activities using the Informant Questionnaire on Cognitive Decline in the Elderly (Jorm, 1994). None of the MCI patients were reported by informants to have any observable changes in usual daily living activities.

Memory impairments in MCI and AD patients were quantified using the word-list learning task from the Consortium to Establish a Registry for Alzheimer's Disease (CERAD) test battery (Morris et al., 1989) and the Logical Memory II subscale of the Wechsler Memory Scale-Revised (Wechsler, 1987). Memory and other test scores were considered abnormal if they fell two standard deviations or more below relevant norms based on age and education. As shown in Table 2, MCI patients generally displayed poorer memory performance than controls, and $\mathrm{AD}$ patients generally displayed poorer memory performance than MCI patients.

In addition to memory impairments, the diagnosis of $\mathrm{AD}$ requires impairment in at least one additional cognitive domain. Standardized neuropsychological tests used to assess impairments in cognitive domains other than declarative memory included the Mini-Mental State Examination (Folstein, Folstein, \& McHugh, 1975), the Category Fluency Test (Morris et al., 1989), the Constructions subtest of the CERAD test battery (Morris et al., 1989), Trail Making Test—Parts A and B (Reitan, 1992), and the Boston Naming Test (Kaplan, Goodglass, \& Weintraub, 1983). As shown in Table 1, each AD patient exhibited significant deficits for age and level of education in at least one cognitive domain other than declarative memory except for AD Patient 8, who was diagnosed with $\mathrm{AD}$ because of the presence of symptoms in daily living, such as getting lost while driving. In addition to low memory scores, two of our MCI participants (MCI Patients 3 and 8) also had low scores on the Boston Naming Test for age

Table 1

Individual Patient and Group Results From General Neuropsychological Testing

\begin{tabular}{|c|c|c|c|c|c|c|}
\hline \multirow[b]{2}{*}{ Patient/group } & \multirow[b]{2}{*}{ MMSE } & \multirow[b]{2}{*}{$\begin{array}{c}\text { Category } \\
\text { Fluency Test }\end{array}$} & \multirow[b]{2}{*}{$\begin{array}{c}\text { Constructions } \\
\text { test }\end{array}$} & \multicolumn{2}{|c|}{ Trail Making Test } & \multirow[b]{2}{*}{$\begin{array}{c}\text { Boston } \\
\text { Naming Test }\end{array}$} \\
\hline & & & & Part A & Part B & \\
\hline Control group & $29.4(0.3)$ & $26.9(3.4)$ & $10.4(0.2)$ & $41.3(5.1)$ & $107.1(22.9)$ & $57.9(0.8)$ \\
\hline MCI Patient 1 & 28 & 12 & 9 & 51 & 141 & 55 \\
\hline MCI Patient 2 & 29 & 22 & 9 & 82 & 138 & 60 \\
\hline MCI Patient 3 & 27 & 15 & 10 & 54 & 116 & $37^{\mathrm{a}}$ \\
\hline MCI Patient 4 & 29 & 17 & 10 & 46 & 102 & 56 \\
\hline MCI Patient 5 & 27 & 13 & 10 & 39 & 84 & 55 \\
\hline MCI Patient 6 & 30 & 28 & 11 & 36 & 104 & 60 \\
\hline MCI Patient 7 & 28 & 17 & 8 & 56 & 108 & 52 \\
\hline MCI Patient 8 & 28 & 11 & 10 & 42 & 208 & $36^{\mathrm{a}}$ \\
\hline MCI group & $28.3(0.4)$ & $16.9(2.0)$ & $9.8(0.4)$ & $50.8(5.1)$ & $125.1(13.6)$ & $51.0(3.3)$ \\
\hline AD Patient 1 & $23^{\mathrm{a}}$ & 11 & 10 & 67 & 117 & $27^{\mathrm{a}}$ \\
\hline AD Patient 2 & $24^{\mathrm{a}}$ & $9^{\mathrm{a}}$ & 10 & & & $22^{\mathrm{a}}$ \\
\hline AD Patient 3 & $26^{\mathrm{a}}$ & 13 & 10 & & & 58 \\
\hline AD Patient 4 & $26^{\mathrm{a}}$ & 17 & 10 & 45 & 152 & 52 \\
\hline AD Patient 5 & $24^{\mathrm{a}}$ & 17 & 10 & 45 & 189 & 55 \\
\hline AD Patient 6 & 29 & 15 & $8^{\mathrm{a}}$ & 31 & $\mathrm{OT}^{\mathrm{a}}$ & 53 \\
\hline AD Patient 7 & $25^{\mathrm{a}}$ & 10 & $8^{\mathrm{a}}$ & 39 & $275^{\mathrm{a}}$ & 47 \\
\hline AD Patient 8 & 28 & 10 & 11 & 66 & 111 & 60 \\
\hline AD group & $25.6(0.7)$ & $14.9(2.0)$ & $9.6(0.4)$ & $48.8(6.0)$ & $168.8(30.0)$ & $46.8(5.0)$ \\
\hline
\end{tabular}

Note. Numbers in parentheses are standard errors of the mean. Maximum scores: MMSE $=30$; Category

Fluency Test $=11$; Constructions test $=11$; Trail Making Test-Parts A and B $=0$ (best score $=0$ ); Boston

Naming Test $=60$. MMSE $=$ Mini-Mental State Examination; OT $=$ timed out.

${ }^{\text {a }}$ Score considered impaired compared with the norms for individuals of the same sex, age, and education level. 
Table 2

Scores From Standardized Memory Tests for Healthy Older Individuals (Controls), Patients With Mild Cognitive Impairment (MCI), and Patients With Alzheimer's Disease (AD)

\begin{tabular}{|c|c|c|c|c|c|c|c|c|c|c|}
\hline \multirow{2}{*}{$\begin{array}{l}\text { Patient } \\
\text { group }\end{array}$} & \multicolumn{2}{|c|}{$\begin{array}{l}\text { CERAD Word } \\
\text { List Trials } 1-3\end{array}$} & \multicolumn{2}{|c|}{$\begin{array}{c}\text { CERAD } \\
\text { Delayed Recall }\end{array}$} & \multicolumn{2}{|c|}{$\begin{array}{l}\text { CERAD } \\
\text { Recognition } \\
\text { hits }\end{array}$} & \multicolumn{2}{|c|}{$\begin{array}{l}\text { CERAD } \\
\text { Recognition } \\
\text { false alarms }\end{array}$} & \multicolumn{2}{|c|}{$\begin{array}{l}\text { WMS-R } \\
\text { Logical } \\
\text { Memory II }\end{array}$} \\
\hline & Score & SEM & Score & SEM & Score & SEM & Score & SEM & Score & SEM \\
\hline Control & 26.4 & 0.9 & 8.8 & 0.6 & 10.0 & 0.0 & 0.0 & 0.0 & 34.1 & 1.9 \\
\hline MCI & 21.5 & 1.5 & 5.8 & 1.2 & 8.8 & 0.9 & 0.3 & 0.3 & 20.8 & 4.7 \\
\hline $\mathrm{AD}$ & 13.8 & 2.3 & 2.5 & 0.4 & 9.0 & 0.6 & 1.6 & 0.7 & 3.8 & 1.5 \\
\hline
\end{tabular}

Note. $\mathrm{CERAD}=$ Consortium to Establish a Registry for Alzheimer's Disease test battery; WMS-R = Wechsler Memory Scale-Revised; SEM = standard error of the mean.

and education. However, these individuals did not fit $D S M-I V$ criteria for dementia because in neither case was there evidence of a change in functioning in daily living activities. All AD patients had a clinical dementia rating (CDR) of 1 (mild; Morris, 1993), whereas all MCI patients had a CDR of 0 or 0.5 .

The mean age of the AD patients was 71.9 years (range: $60-86$ ), and the mean age of the MCI patients was 76.4 years (range: 61-84). On average, AD patients had completed 14.1 years of education (range: 10-18), and MCI patients had completed 17.0 years of education (range: 9-20). AD and MCI groups did not differ in average age, $t(14)=1.07, p>.3$, or years of education, $t(14)=1.76, p>.1$.

Three of the $8 \mathrm{MCI}$ patients were taking donepezil prescribed by their primary care physicians. Seven of the $8 \mathrm{AD}$ patients were taking either donepezil (4), rivastigmine (2), or galantamine (1).

Healthy older individuals ( 8 women) were recruited as cognitively normal control participants. Mean age ( 81.0 years; range: $70-94)$ and years of education (16.4 years; range: 14-20) did not differ significantly from those in the MCI group: age, $t(14)=1.29, p>.2$; education, $t(14)=0.43$, $p>$.6. The control group was also matched in years of education to the AD group, $t(14)=1.79, p>.09$, and was slightly older than the AD group, $t(14)=2.21, p>.04$.

Thirty-six young adults (15 men, 21 women) from an introductory psychology class at Northwestern University also participated in exchange for course credit.

Prior to data collection, approval for human participants research was obtained from the Institutional Review Board at Northwestern University, and informed consent was obtained from all participants.

\section{Materials}

Four highly similar versions of each of 24 nameable objects were used as stimuli. Sample stimuli are shown in Figure 1. Half of the objects were naturally occurring, and half were human artifacts. Target objects were
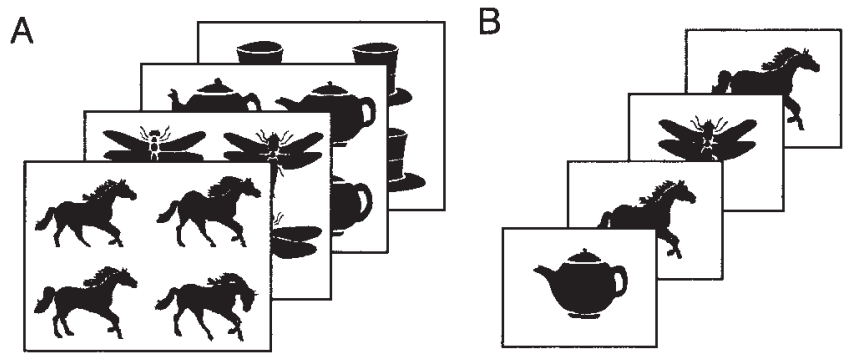

Figure 1. Test format for (A) forced-choice recognition and (B) yes-no recognition. randomly selected from each group of four similar pictures. Stimuli were divided into two sets, and a discrimination task conducted by Holdstock and colleagues (2002) ensured that the similarity of targets and their corresponding foils did not differ between the two sets.

\section{Procedure}

Patients and controls. Each participant completed a forced-choice recognition test and a yes-no recognition test. The order of the tests and the set of objects used in each test were counterbalanced across participants. A learning phase and corresponding testing phase were completed for the first test, followed by a short break. Subsequently, learning and testing phases for the second test were completed with a different set of objects.

Prior to each test, participants completed a practice run to become accustomed to the learning and test procedure. The practice procedure was identical to the main test except that participants studied 6 objects instead of 12 and the practice tests contained half as many test items. Objects were of the same style as in the main tests with comparable target-foil similarity. Thus, in addition to being instructed to pay close attention to details during the learning phase, the practice runs also alerted participants to the fact that they should attend to visual details of each object during the learning phase to make the memory judgments required in the test phase.

The procedure in the learning phase was identical for the two tests. Participants viewed each of 12 target objects twice at a rate of $3 \mathrm{~s}$ per object. Stimuli were presented on two sheets of letter-size paper with six objects on each sheet. Attention was drawn to individual objects by placing a cardboard mask over each page such that only one object was visible through the viewing window at a time. For the first presentation, participants decided whether each object was natural or man made. After judgments for all 12 objects had been made, the objects were presented for a second time. For the second presentation, participants were instructed to study the details of each object. Immediately following completion of the object presentations, participants completed math problems for $45 \mathrm{~s}$, which minimized rehearsal of the recently viewed objects. Testing began immediately following completion of the math problems. Mean retention delay between the second presentation of a stimulus in the learning phase and its appearance in the test phase was approximately $2.5 \mathrm{~min}$ for each test.

On each trial of the forced-choice recognition test, the participant viewed a target object and three corresponding foils evenly distributed on a page (see Figure 1A). There were 12 test trials, randomly ordered. Participants were asked to select the target on each page.

On each trial of the yes-no recognition test, test items were presented one at a time on small cards (see Figure 1B). Participants were asked to respond "yes" if they thought the object was one of the target objects and "no" if they did not. Test objects were presented in a random order and included the 12 target objects and 36 foils (three corresponding to each of the 12 target objects). Twelve additional trials were randomly intermixed among the 48 test trials to minimize the possibility that decisions about 
each test object were influenced by decisions made about previous test objects. Four target objects were presented one additional time, and four different target objects were presented two additional times, for a total of 60 test trials. Only responses from the first presentation of each target were included in subsequent analyses.

Young adults. The procedure described above was slightly modified for young adults to accommodate a 1- or 2-week delay between learning and testing phases although stimuli were identical. Participants first completed both practice runs. They then completed the learning phase for both tests, viewing two sets of objects and completing math problems for $45 \mathrm{~s}$ immediately after the second presentation of each set of objects.

Twelve of the participants were assigned to the 3-min-delay condition. For these participants, the yes-no and forced-choice recognition tests were administered immediately following the second set of math problems. Test trials in the first test corresponded to target stimuli studied in the first learning phase (but in random order). The average retention delay for each test was approximately $3 \mathrm{~min}$. Twelve of the remaining participants were assigned to the 1-week-delay condition and returned 1 week later, and the final 12 participants were assigned to the 2-week-delay condition and returned 2 weeks later. The two tests were administered in the order of corresponding learning phases. Furthermore, the order of the two tests and the set of objects used in each test were counterbalanced across participants in all three delay conditions.

\section{Results}

Raw accuracy scores across the two memory tests were based on quite different chance performance rates. To obtain performance measures affording straightforward comparisons, hit and falsealarm rates for the yes-no test and the hit rate for the forced-choice test were used to calculate estimates of recognition sensitivity $\left(d^{\prime}\right)$ for each participant (Macmillan \& Creelman, 1991). Because the calculation of sensitivity requires that raw proportions be transformed into $z$ scores, all observed proportions were corrected by adding 0.5 to each frequency and dividing by $N+1$ (where $N=$ number of trials), as recommended by Snodgrass and Corwin (1988). This correction has minimal effects for proportions near 0.5. Table 3 shows hit rates and false-alarm rates (without this correction), along with estimated sensitivity $\left(d^{\prime}\right)$, for patients and controls.

\section{Patients}

Compared with performance in the control group, recognition sensitivity in the MCI group appeared diminished on the yes-no test but not on the forced-choice test (see Figure 2A). A $2 \times 2$ analysis of variance (ANOVA) was conducted using $d^{\prime}$ estimates as the dependent variable, test format (yes-no or forced choice) as the within-subjects independent variable, and participant group
(MCI or control) as the between-subjects independent variable. For this and all subsequently reported ANOVAs, the effect size for each main effect and interaction was also measured by calculating eta-squared $\left(\eta^{2}\right)$. Effect size can be interpreted according to the standards defined by Cohen (1988) in which $\eta^{2}=.2$ is small, $\eta^{2}=$ .5 is medium, and $\eta^{2}=.8$ is large. The interaction between test format and participant group was significant, $F(1,14)=7.53$, $M S E=0.14, p<.05, \eta^{2}=.35$, and post hoc $t$ tests showed a significant impairment for MCI patients compared with controls on the yes-no test, $t(14)=3.40, p<.01$, but not on the forced-choice test, $t(14)<1$. Overall differences as a function of group and test format were nonsignificant, $F(1,14)=3.73, M S E=0.23, p>.07$, $\eta^{2}=.21$, and $F(1,14)<1, \eta^{2}=.0003$, respectively.

\section{AD Patients}

Compared with performance in the control group, recognition sensitivity in the AD group was lower on both tests (see Figure 2B). As above, $d^{\prime}$ estimates were submitted to a $2 \times 2$ ANOVA. A significant main effect of group, $F(1,14)=39.5, M S E=0.10$, $p<.001, \eta^{2}=.74$, confirmed that memory in AD patients was impaired. The interaction between test format and group was marginal, $F(1,14)=4.52, M S E=0.17, p<.06, \eta^{2}=.24$, suggesting that the impairment varied depending on test format. Post hoc $t$ tests showed that the AD impairment was present for both the yes-no test, $t(14)=5.34, p<.0001$, and the forcedchoice test, $t(14)=2.20, p<.05$, but the impairment was numerically larger in the yes-no test. The main effect of test format was nonsignificant, $F(1,14)<1, \eta^{2}=.02$.

\section{Comparisons Between Patient Groups}

To assess the relative impairments in yes-no and forced-choice recognition between $\mathrm{MCI}$ and $\mathrm{AD}$ patients, the $d^{\prime}$ estimate calculated for each patient was subtracted from the mean $d^{\prime}$ estimate for the control participants to obtain an impairment estimate for each patient for each test (yes-no and forced choice). A $2 \times 2$ ANOVA was conducted using impairment estimates as the dependent variable, test format as the within-subjects independent variable, and patient group (MCI or AD) as the between-subjects independent variable. Results are displayed in Figure 3. The main effect of patient group was significant, $F(1,14)=6.17, M S E=0.19, p<$ $.05, \eta^{2}=.31$, demonstrating that memory impairments were greater for $\mathrm{AD}$ patients than for MCI patients. The main effect of test format was also significant, $F(1,14)=21.4, M S E=0.17, p<$ $.001, \eta^{2}=.60$, indicating that patients in both groups were more

Table 3

Recognition Results for Healthy Older Individuals (Controls), Patients With Mild Cognitive Impairment (MCI), and Patients With Alzheimer's Disease (AD)

\begin{tabular}{|c|c|c|c|c|c|c|c|c|c|c|}
\hline \multirow{2}{*}{$\begin{array}{l}\text { Patient } \\
\text { group }\end{array}$} & \multicolumn{6}{|c|}{ Yes-no recognition } & \multicolumn{4}{|c|}{ Forced-choice recognition } \\
\hline & Hit rate & SEM & FA rate & SEM & $d^{\prime}$ & SEM & Hit rate & SEM & $d^{\prime}$ & SEM \\
\hline Control & .85 & .05 & .41 & .05 & 1.30 & .15 & .52 & .05 & 0.90 & .19 \\
\hline MCI & .74 & .05 & .51 & .04 & 0.60 & .14 & .53 & .06 & 0.93 & .19 \\
\hline $\mathrm{AD}$ & .76 & .08 & .68 & .07 & 0.26 & .12 & .39 & .05 & 0.50 & .14 \\
\hline
\end{tabular}

Note. $\quad$ FA rate $=$ false-alarm rate; $d^{\prime}=$ estimated sensitivity; $S E M=$ standard error of the mean. 


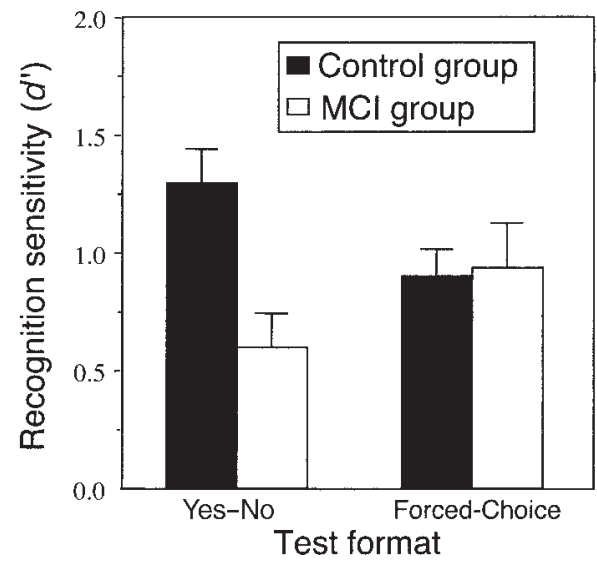

B

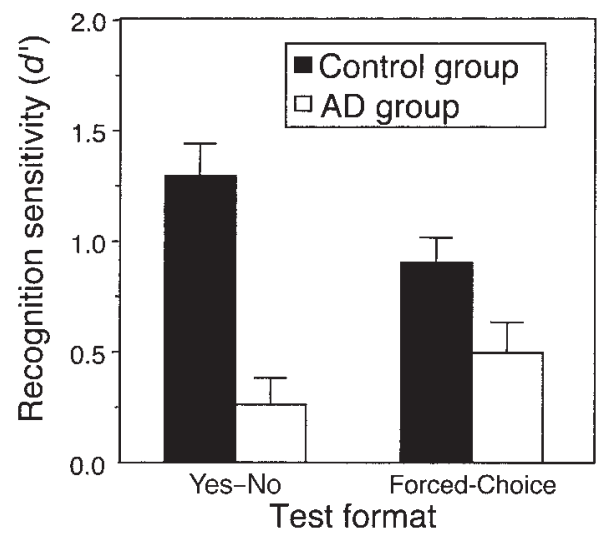

Figure 2. Recognition sensitivity $\left(d^{\prime}\right)$ plotted as a function of test format (yes-no or forced choice) for patients and healthy older controls. A: control group versus $\mathrm{MCI}$ group. B: control group versus $\mathrm{AD}$ group. Bars indicate standard errors of the mean. $\mathrm{AD}=$ Alzheimer's disease; $\mathrm{MCI}=$ mild cognitive impairment.

impaired in yes-no recognition than in forced-choice recognition. The interaction between patient group and test format was not significant, $F(1,14)<1, \eta^{2}=.003$.

\section{Response Bias}

According to signal-detection theory (Green \& Swets, 1966; Macmillan \& Creelman, 1991), the use of a forced-choice-test format eliminates any response bias, which can be thought of as the nonspecific tendency to endorse stimuli as belonging to one category or the other. In yes-no tests, the $d^{\prime}$ estimate of sensitivity provides a measure of response accuracy independent of any existing response bias given that the variances of the underlying target and foil memory strength distributions are equivalent. However, in recognition memory experiments, this assumption is not typically warranted (e.g., Ratcliff, Sheu, \& Gronlund, 1992; Yonelinas, 1994). When the variances of the underlying target and foil distributions are unequal, $d^{\prime}$ estimates vary with response bias. Specifically, $d^{\prime}$ estimates increase as the strictness of the response criterion increases (Kroll, Yonelinas, Dobbins, \& Frederick, 2002). Thus, it is possible that differences in yes-no $d^{\prime}$ calculated for patients and controls in the present experiment may have been distorted by differences in response bias across participant groups. Therefore, we also estimated response bias $(c)$ in the manner described by Macmillan and Creelman (1991) for each participant for the yes-no test. All participant groups exhibited a bias to respond "yes" $(\mathrm{MCI}$ group $=-0.32, \mathrm{AD}$ group $=-0.65$, control group $=-0.43$; where negative values indicate a bias to respond "yes," positive values indicate a bias to respond "no," and 0 indicates no bias to respond "yes" or "no"). A one-way ANOVA was conducted using yes-no bias measures as the dependent variable and group as the between-subjects independent variable. No main effect of group was observed, $F(2,21)=1.07$, $M S E=0.21, p>.3, \eta^{2}=.09$. Post hoc $t$ tests revealed that neither the $\mathrm{AD}$ group, $t(14)=0.83, p>.4$, nor the MCI group, $t(14)=0.61, p>.5$, had a response bias significantly different from that in the control group. Additionally, response bias did not differ between the $\mathrm{AD}$ group and the MCI group, $t(14)=1.35, p>$ .1. Thus, it is likely that response bias in the yes-no test did not distort the analyses of $d^{\prime}$ estimates across the three groups.

\section{Young Adults}

To directly compare memory performance on yes-no versus forced-choice recognition tests as a function of normal memory decay, the analysis procedures described above were used to estimate $d^{\prime}$ based on hit and false-alarm rates (yes-no test) and the proportion of correct responses (forced-choice test) observed in young adults. In both tests, recognition after a 3-min delay was much better than after either a 1-week or 2-week delay, as shown in Figure 4. An ANOVA was conducted with test format (yes-no or forced choice) as the within-subjects independent variable and delay (short, 1-week, or 2-week) as the between-subjects independent variable. The main effect of delay was significant, $F(2$, $33)=19.9, M S E=0.37, p<.001, \eta^{2}=.55$, and post hoc $t$ tests confirmed that 3-min-delay sensitivity (1.65) was higher than 1 -week-delay sensitivity $(0.95), t(22)=3.93, p<.001$, and that 1 -week-delay sensitivity was higher than 2 -week-delay sensitivity (0.56), $t(14)=2.40, p<.05$. Neither the main effect of test

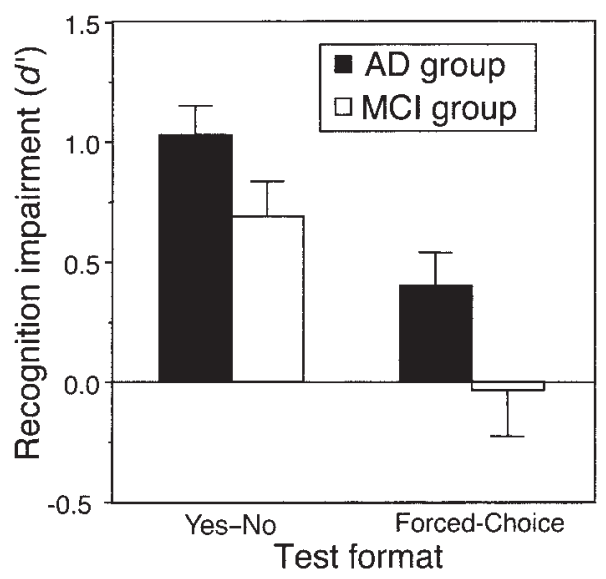

Figure 3. Recognition impairment estimates $\left(M\right.$ control $d^{\prime}-$ patient $\left.d^{\prime}\right)$ plotted as a function of test format (yes-no or forced choice) and patient group $(\mathrm{AD}$ or $\mathrm{MCI})$. Bars indicate standard errors of the mean. $\mathrm{AD}=$ Alzheimer's disease; $\mathrm{MCI}=$ mild cognitive impairment. 


\section{- 3-min delay \\ $\square$ 1-week delay $\square$ 2-week delay}

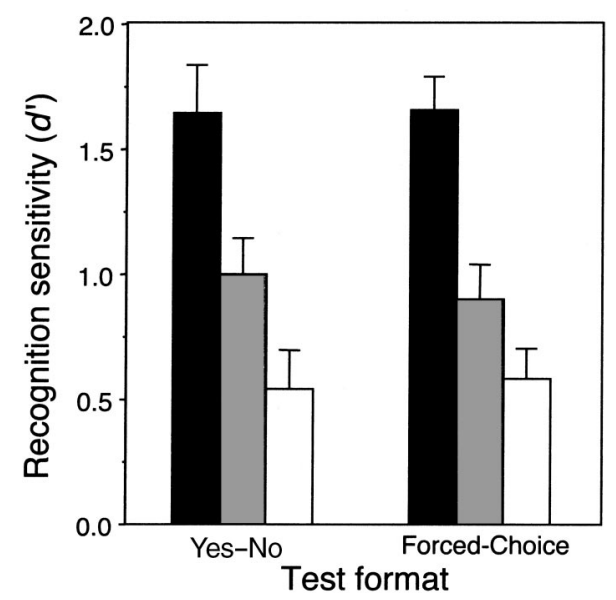

Figure 4. Recognition sensitivity $\left(d^{\prime}\right)$ for young adults plotted as a function of test format (yes-no or forced choice) and retention delay (3 min, 1 week, or 2 weeks). Bars indicate standard errors of the mean.

format, $F(1,33)<1, \eta^{2}=.0009$, nor the interaction between test format and delay, $F(2,33)<1, \eta^{2}=.01$, approached significance.

\section{Discussion}

Distinctive patterns of memory impairment were observed in patients with $\mathrm{AD}$ and MCI. A novel glimpse into the memory dysfunctions experienced by these patients was obtained by using two very difficult recognition tests, one designed to be differentially sensitive to recollection (the yes-no test) and the other to familiarity (the forced-choice test). Recognition impairments in $\mathrm{AD}$ patients were clearly evident in both tests (despite the slightly older mean age of the controls) and were more severe than in MCI patients. Furthermore, AD and MCI patients exhibited larger impairments on the yes-no test compared with the forced-choice test. Recognition impairments in MCI patients were found only in the yes-no test. The finding that forced-choice recognition performance did not differ between the MCI group and the age- and education-matched control group is striking, especially given the high difficulty of the test. Preserved memory on a test of low general difficulty could be ascribed to the mild nature of the memory impairments. That sort of explanation is not appropriate here.

To evaluate these findings, one must carefully consider the relative difficulty of the yes-no and forced-choice tests. Preserved recognition on the forced-choice test might be expected if that test was generally less difficult than the yes-no test. Holdstock and colleagues (2002) directly examined possible differences in difficulty across the same two tests in a group of control participants. Difficulty scores were calculated as a percentage that reflected where the mean performance level fell between chance and a perfect score for each test. This analysis indicated that the two tests have approximately the same level of difficulty. Moreover, the slightly (but not significantly) higher yes-no performance com- pared with forced-choice performance of the control group in the present experiment reinforces the findings from Holdstock and colleagues and suggests that relatively preserved forced-choice performance of $\mathrm{AD}$ and MCI patients was not a result of a relatively easier forced-choice than yes-no test. The two tests do make different demands on memory processing, but these differences are not simply a confound with difficulty. ${ }^{1}$ Instead, the present results indicate a disproportionate disruption of recollection compared with familiarity in MCI and AD. In keeping with this conclusion, memory impairments for patients relative to controls were larger in the yes-no recognition test than in the forcedchoice recognition test.

The present results are consistent with previous suggestions that recollection may be more disrupted than familiarity in AD (Bartok et al., 1997; Budson, Daffner, Desikan, \& Schacter, 2000; Dalla Barba, 1997; Gallo, Sullivan, Daffner, Schacter, \& Budson, 2004; Knight, 1998; Koivisto, Portin, Seinela, \& Rinne, 1998; Tendolkar et al., 1999). No previous studies have examined the efficacy of recollection and familiarity in $\mathrm{MCI}$, and our findings reveal something quite remarkable. Although recollection was impaired in these patients, familiarity-based recognition was completely intact despite the memory deficits present in MCI.

It should be noted that the relatively lenient criteria exhibited in all three participant groups on the yes-no test may have lowered estimates of recognition sensitivity for all groups on this test, compromising straightforward comparisons with sensitivity estimates for the forced-choice test (Kroll et al., 2002). However, this possibility does not impact the most interesting findings reported here, namely, that the relative difference in memory performance observed in $\mathrm{AD}$ and $\mathrm{MCI}$ patient groups compared with the control group was smaller in the forced-choice test than in the yes-no test.

These neuropsychological findings might be considered less meaningful if the pattern of disproportionate decline in yes-no

\footnotetext{
${ }^{1}$ Another difference between the yes-no and forced-choice tests is the time required to take each test. In the forced-choice test, memory is tested for one target and three foils on each trial. In contrast, each trial in the yes-no test concerns memory for one item (either a target or a foil). Consequently, there are many more trials in the yes-no test, the test takes longer to complete, and the time from study to test is longer on average. Also, more interference may build up during the yes-no test phase given the mixed sequence of trials corresponding to different targets. Further experiments are needed to investigate the relevance of these various dimensions whereby the two tests differ. To explore possible effects of the extended duration of the yes-no test, we compared performance between the first and second halves. Performance did not differ significantly as a function of test half (corresponding $d^{\prime}$ scores for the AD group: 0.52 and 0.49; for the MCI group: 0.97 and 0.57; and for the control group: 1.29 and 1.39). Both patient groups showed impaired performance relative to the control group in the second half, whereas only the AD group showed a clear impairment in the first half, thus raising the possibility that the apparent impairment in the MCI group required a lengthy yes-no test. However, we collected additional data from 28 participants and observed significant impairments in MCI in both halves ( $d^{\prime}$ scores by test half for the new MCI group [ $N=14]$ : 0.62 and 0.67 ; and for the new control group $[N=14] ; 1.37$ and 1.21). The yes-no impairment in MCI patients thus appears to be reliable. Interpretations must take into account test length and possible test-phase interference, but determining exactly how performance might change over the course of the test would require balanced tests specifically designed for this purpose.
} 
compared with forced-choice recognition was also observed in situations when memory traces are weak without any accompanying neuropathology, such as with the simple passage of time. To address this issue, we tested recognition in young adults using the same two tests at three different delays ( 3 min, 1 week, 2 weeks). Although some evidence suggests that familiarity may decline more rapidly than recollection at very short delays ( $<1 \mathrm{~min})$, other evidence suggests that at longer delays $(>1 \mathrm{~min})$, recollection and familiarity decline at comparable rates (see Yonelinas, 2002). A disproportionate decline for yes-no compared with forced-choice recognition in healthy young adults would suggest that the dissociation observed in MCI and AD patients using these same two tests may not reflect differences in the efficacy of recollection and familiarity per se. Rather, it is conceivable that these differences merely reflect trivial differences inherent in the different tests.

Our results indicated that both forced-choice recognition and yes-no recognition were significantly lower after a 1-week delay compared with a 3-min delay and that these declines were equivalent for the two tests. Furthermore, after a 2-week delay, when memory performance on both tests had declined to a level that was lower than memory performance observed on either test for MCI patients, the declines in yes-no and forced-choice recognition were still equivalent. Therefore, we conclude that the dissociation observed between yes-no and forced-choice recognition in AD and MCI is not a result of uninteresting differences inherent in the two tests. Disproportionate deficits in yes-no recognition do not merely reflect weak memory but rather are a consequence of the special character of the memory disorder in these patient groups.

Holdstock and colleagues (2002) demonstrated that yes-no, but not forced-choice, recognition was impaired in a patient with focal hippocampal damage. Accordingly, they proposed that (a) performance on the yes-no recognition test depends centrally on recollection, which requires the integrity of the hippocampus, and (b) performance on the forced-choice recognition test depends centrally on familiarity, which requires the integrity of MTC (entorhinal, perirhinal, and parahippocampal cortices) but does not require the integrity of the hippocampus. These postulates receive additional support from the present findings that $\mathrm{AD}$ and $\mathrm{MCI}$ patients were impaired in yes-no recognition whereas only AD patients were impaired in forced-choice recognition. Although we did not obtain independent verification that the yes-no and forcedchoice tests differentially relied on recollection and familiarity, respectively, this interpretation is consistent with the results obtained by Holdstock and colleagues and also with simulation data from computational models of hippocampus and MTC developed by Norman and O'Reilly (2003). Further research is nevertheless needed to more closely tie the phenomena of recollection and familiarity to recognition performance in these circumstances.

The present findings indicate that forced-choice recognition does not require healthy hippocampal-entorhinal tissue. Indeed, given that forced-choice performance was normal in MCI, familiarity must presumably be sustained by virtue of neural tissue that remained functional in these individuals but that becomes compromised with the progression of $\mathrm{AD}$. Clear neuropathological signs of deterioration are generally evident in hippocampal and entorhinal regions in MCI (e.g., Du et al., 2001; Guillozet et al., 2003), as well as in a region denoted by the term transentorhinal, which is transitional cortex at the border of entorhinal and perirhinal regions. We therefore speculate that familiarity signals in MCI patients relied on contributions from one or more brain regions that remained free of significant pathology or from one or more brain regions that may have been partially damaged but that continued to support effective familiarity-based recognition.

One possible explanation for the relative preservation of forcedchoice recognition in $\mathrm{AD}$ and $\mathrm{MCI}$ emphasizes perirhinal cortex, as shown schematically in Figure 5A. The key postulate is that perirhinal cortex can produce a familiarity signal sufficient for normal recognition on the forced-choice test with highly similar foils, which is consistent with other evidence suggesting that perirhinal cortex supports familiarity (Brown \& Xiang, 1998; Davachi et al., 2003; Henson et al., 2003; Ranganath et al., 2004). However, the present data would cast doubt on a perirhinal proposal if this region were subject to severe dysfunction in MCI; further evidence on this point from $\mathrm{AD}$ and MCI patients is required given that even subtle pathology could have significant

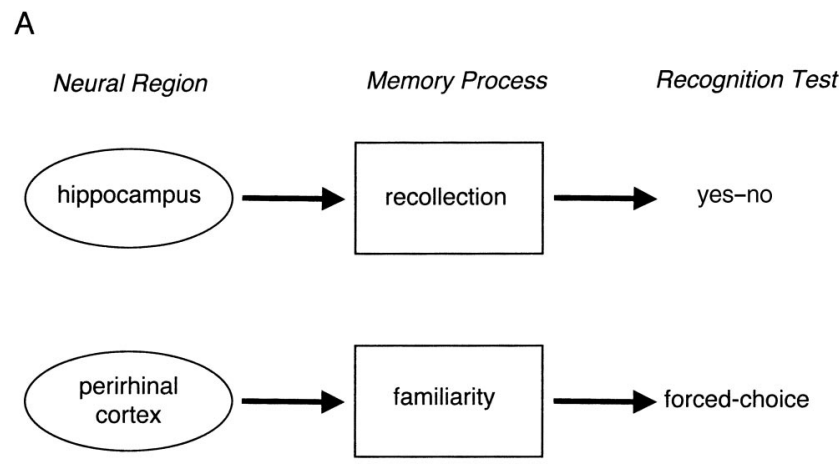

B Neural Region Memory Process Recognition Test

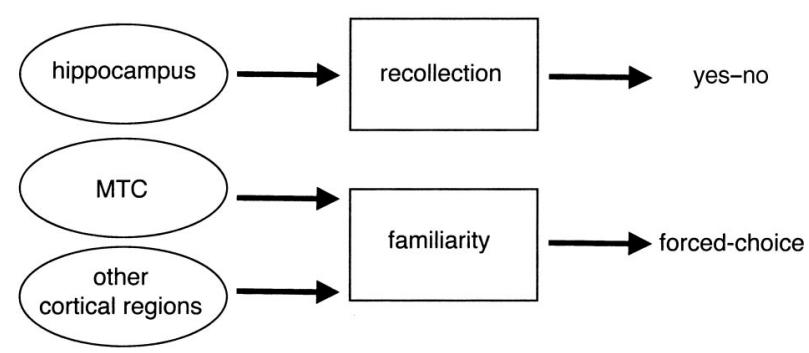

Figure 5. Schematic diagrams showing the hypothetical role of recollection and familiarity in two types of recognition tests with highly similar targets and foils and associated brain-behavior relationships. A: One simple hypothesis is that perirhinal cortex makes a critical contribution to familiarity, whereas a hippocampal contribution is critical to recollection. In turn, yes-no recognition performance may depend disproportionately on recollection, whereas forced-choice recognition performance may depend disproportionately on familiarity. For simplicity, the schematic diagrams do not show all relevant brain regions (e.g., hippocampal processing is important for recollection only together with contributions from other brain regions). Likewise, diagrams show only single arrows to indicate the disproportionate contributions (e.g., omitting reference to the possibility that recollection can contribute to forced-choice recognition performance). B: Another hypothesis describes a more complex set of relationships. Multiple regions within medial temporal cortex (MTC), as well as other cortical regions and perhaps the hippocampus, may contribute to familiarity. 
functional consequences. Indeed, volumetric MRI results indicate that perirhinal cortex exhibits significant atrophy in AD, although not as severe as in entorhinal cortex (Juottonen et al., 1998). Some perirhinal atrophy may thus be present in MCI patients as well, but perhaps it is insufficient to impair familiarity-based recognition.

Specific MTC regions were initially defined in monkey brains, and these regions have subsequently been identified in human brains, although not without some controversy (Amaral, 1999; Insausti et al., 1998). For example, presumptive perirhinal cortex in humans may actually correspond to what has been anatomically defined by some investigators as transentorhinal cortex, which is the site of maximal neurofibrillary pathology in normal aging, MCI, and AD (Braak \& Braak, 1991; Guillozet et al., 2003). Clear distinctions between perirhinal and transentorhinal areas in humans are thus needed before strong conclusions about the extent of perirhinal damage in AD and MCI can be drawn.

An alternative to the perirhinal hypothesis is that familiarity signals arise from many medial temporal regions. These signals may become less effective when the impact of disease reaches a certain critical threshold, as in the AD group. However, partial damage that has not reached this critical threshold, as in the MCI group, may be sufficient to support effective familiarity signals. An intriguing possibility is that partial hippocampal damage can disrupt recollection, whereas remaining hippocampal tissue could still effectively support familiarity. Future research should be aimed at achieving a more complete assessment of familiarity processes in patients with MCI, such as through remember-know and receiver operating characteristic methods, to determine whether the efficacy of familiarity in MCI remains high when testing demands vary. Our results suggest that further insights into the anatomy of memory may result from linking specific sites of Alzheimer-related brain damage with declines in familiarity-based recognition assessed using the present paradigm.

Indeed, one limitation of the current experiment is the unavailability of detailed pathological analyses for each MCI and AD patient in our sample. In general, neuropathological investigations of MCI and AD reliably detect medial temporal damage in individuals with these clinical diagnoses (Price \& Morris, 1999; Troncoso, Martin, Dal Forno, \& Kawas, 1996). Furthermore, the extent of such damage has been shown to vary with the level of memory impairment. For example, Köhler and colleagues (1998) demonstrated that hippocampal volume in patients with $\mathrm{AD}$ positively correlated with performance on an auditory-verbal list-learning recall task and that parahippocampal gyrus volume positively correlated with performance on a nonverbal visual-recall task. Investigations of the extent to which pathology in specific regions correlates with memory performance in MCI and AD patients are a promising area for future research-the distinct roles of recollection and familiarity should be taken into account in such work.

Our results are also consistent with the idea that structures beyond MTC may contribute to familiarity, as shown schematically in Figure 5B. In addition to MTC structures, areas of visual cortex essential for visual object perception and recognition may play some role in mediating processes that underlie familiarity for visual stimuli. These areas presumably remain intact in MCI but begin to deteriorate as pathology spreads to neocortex with the progression of $\mathrm{AD}$. In the present group of $\mathrm{AD}$ patients, pathology may have progressed to these other neocortical areas, leading to some impairment in forced-choice recognition. Such damage could account for the forced-choice recognition deficits observed in the $\mathrm{AD}$ patients but not in the MCI patients, whereas damage to hippocampus and entorhinal cortex could impair yes-no recognition in both $\mathrm{AD}$ and MCI.

In addition to memory deficits, individuals with $\mathrm{AD}$ also exhibit perceptual deficits (e.g., Cronin-Golomb et al., 1991). Visuoperceptual deficits may have reduced the ability of $\mathrm{AD}$ patients to effectively encode and store sufficient detail to successfully complete the recognition tasks in this experiment. These perceptual deficits may have contributed to the lower memory performance of AD patients compared with controls on both the yes-no and forced-choice tests. However, perceptual deficits would equally impair performance across the two tests. The observed dissociation between impairments in yes-no and forced-choice recognition in $\mathrm{AD}$ patients is thus most likely a result of deficient memory processing rather than visuoperceptual deficits.

The relative preservation of familiarity in $\mathrm{AD}$ and MCI demonstrated here is also noteworthy for ramifications beyond laboratory studies of memory. Understanding the nature of these memory disorders with reference to distinct roles of recollection and familiarity may have important implications for treating individuals who suffer in their daily lives from memory lapses. In particular, therapeutic strategies in patients with $\mathrm{AD}$ and $\mathrm{MCI}$ designed to increase dependence on familiarity-based memory in daily living may help to reduce some of the negative consequences resulting from memory decline.

It is also important to note that the concept of MCI is at present somewhat controversial. For instance, it is typically accepted that activities of daily living are preserved in individuals with MCI (Petersen et al., 2001) although recent reports using detailed informant interviews have suggested that nondemented individuals with mild cognitive deficits may have some difficulty with certain daily living activities (Artero, Touchon, \& Ritchie, 2001; Tabert et al., 2002). Furthermore, a diagnosis of MCI can be made on the basis of a variety of cognitive deficits, and further classification of MCI into various subtypes is now emerging (Petersen, 2004). Although, in the present study, we have emphasized memory deficits leading to $\mathrm{AD}$, the category of MCI may encompass more than one disease process, perhaps including factors related to cerebrovascular disease (Galluzzi, Sheu, Zanetti, \& Frisoni, 2005; Roman et al., 2004), and there may be additional MCI subtypes yet to be described.

In conclusion, the present results indicate that in patients with diagnoses of $\mathrm{AD}$ or MCI, familiarity is relatively preserved compared with recollection. Impaired yes-no recognition in AD and $\mathrm{MCI}$ is consistent with prior results suggesting that damage to the hippocampus impairs recollection (e.g., Holdstock et al., 2002). The remarkable findings that forced-choice recognition is relatively intact in $\mathrm{AD}$ and entirely intact in MCI provide insights into the critical neural substrates of familiarity signals. Neural processing that supports familiarity may include contributions from perirhinal cortex, from multiple medial temporal regions that are partially damaged but nonetheless can continue to support familiarity, and/or from structures outside the medial temporal region.

\section{References}

Aggleton, J. P., \& Brown, M. W. (1999). Episodic memory, amnesia, and the hippocampal anterior thalamic axis. Behavioral and Brain Sciences, 22, 425-489. 
Aggleton, J. P., Vann, S. D., Denby, C., Dix, S., Mayes, A. R., Roberts, N., \& Yonelinas, A. P. (2005). Sparing of the familiarity component of recognition memory in a patient with hippocampal pathology. Neuropsychologia, 43, 1810-1823.

Amaral, D. G. (1999). What is where in the medial temporal lobe? Hippocampus, 9, 1-6.

American Psychiatric Association. (1994). Diagnostic and statistical manual of mental disorders (4th ed.). Washington, DC: Author.

Artero, S., Touchon, J., \& Ritchie, K. (2001). Disability and mild cognitive impairment: A longitudinal population-based study. International Journal of Geriatric Psychiatry, 16, 1092-1097.

Baddeley, A., Vargha-Khadem, F., \& Mishkin, M. (2001). Preserved recognition in a case of developmental amnesia: Implications for the acquisition of semantic memory? Journal of Cognitive Neuroscience, 13, 357-369.

Bartok, J. A., Wilson, C. S., Giordani, B., Keys, B. A., Persad, C. C., Foster, N. L., \& Berent, S. (1997). Varying patterns of verbal recall, recognition, and response bias with progression of Alzheimer's disease. Aging, Neuropsychology and Cognition, 4, 266-272.

Bastin, C., \& Van der Linden, M. (2003). The contribution of recollection and familiarity to recognition memory: A study of the effects of test format and aging. Neuropsychology, 17, 14-24.

Bastin, C., Van der Linden, M., Charnallet, A., Denby, C., Montaldi, D., Roberts, N., \& Mayes, A. R. (2004). Dissociation between recall and recognition memory performance in an amnesic patient following carbon monoxide poisoning. NeuroReport, 10, 330-344.

Braak, H., \& Braak, E. (1991). Neuropathological staging of Alzheimerrelated changes. Acta Neuropathologica, 82, 239-259.

Brown, M. W., \& Xiang, J. Z. (1998). Recognition memory: Neuronal substrates of the judgement of prior occurrence. Progress in Neurobiology, 55, 149-189.

Budson, A. E., Daffner, K. R., Desikan, R., \& Schacter, D. L. (2000). When false recognition is unopposed by true recognition: Gist-based memory distortion in Alzheimer's disease. Neuropsychology, 14, 27502765.

Budson, A. E., Desikan, R., Daffner, K. R., \& Schacter, D. L. (2001). Perceptual false recognition in Alzheimer's disease. Neuropsychology, 15, 230-243.

Budson, A. E., Michalska, K. J., Sullivan, A. L., Rentz, D. M., Daffner, K. R., \& Schacter, D. L. (2003). False recognition in Alzheimer's disease: Evidence from categorized pictures. Cognitive and Behavioral Neurology, 16, 16-27.

Cohen, J. (1988). Statistical power analysis for the behavioral sciences (2nd ed.). Hillsdale, NJ: Erlbaum.

Cronin-Golomb, A., Corkin, S., Rizzo, J. F., Cohen, J., Growdon, J. H., \& Banks, K. B. (1991). Visual dysfunction in Alzheimer's disease: Relation to normal aging. Annals of Neurology, 29, 41-52.

Dalla Barba, G. (1997). Recognition memory and recollective experience in Alzheimer's disease. Memory, 5, 657-672.

Davachi, L., Mitchell, J. P., \& Wagner, A. D. (2003). Multiple routes to memory: Distinct medial temporal lobe processes build item and source memories. Proceedings of the National Academy of Sciences, USA, 100, 2157-2162.

Delacourte, A., David, J. P., Sergeant, N., Buee, L., Wattez, A., Vermersch, P., et al. (1999). The biochemical pathway of neurofibrillary degeneration in aging and Alzheimer's disease. Neurology, 52, 1158-1165.

de Leon, M. J., Ferris, S. H., George, A. E., Christman, D. R., Fowler, J. S., Gentes, C., et al. (1983). Positron emission tomographic studies of aging and Alzheimer disease. American Journal of Neuroradiology, 4, $568-$ 571.

Du, A. T., Schuff, N., Amend, D., Laakso, M. P., Hsu, Y. Y., Jagust, W. J., et al. (2001). Magnetic resonance imaging of the entorhinal cortex and hippocampus in mild cognitive impairment and Alzheimer's disease. Journal of Neurology, Neurosurgery, and Psychiatry, 71, 441-447.
Du, A. T., Schuff, N., Kramer, J. H., Ganzer, S., Zhu, X. P., Jagust, W. J., et al. (2004). Higher atrophy rate of entorhinal cortex than hippocampus in AD. Neurology, 62, 422-427.

Eldridge, L. L., Knowlton, B. J., Furmanski, C. S., Bookheimer, S. Y., \& Engel, S. A. (2000). Remembering episodes: A selective role for the hippocampus during retrieval. Nature Neuroscience, 3, 1149-1152.

Folstein, M. F., Folstein, S. E., \& McHugh, P. R. (1975). Mini-mental state: A practical method for grading the state of patients for the clinician. Journal of Psychiatric Research, 12, 189-198.

Foster, N. L., Chase, T. N., Mansi, L., Brooks, R., Fedio, P., Patronas, N. J., \& Di Chiro, G. (1984). Cortical abnormalities in Alzheimer's disease. Annals of Neurology, 16, 649-654.

Friedland, R. P., Jagust, W. J., Huesman, R. H., Koss, E., Knittel, B., Mathis, C. A., et al. (1989). Regional cerebral glucose transport and utilization in Alzheimer's disease. Neurology, 39, 1427-1434.

Gallo, D. A., Sullivan, A. L., Daffner, K. R., Schacter, D. L., \& Budson, A. E. (2004). Associative recognition in Alzheimer's disease: Evidence for impaired recall-to-reject. Neuropsychology, 18, 556-563.

Galluzzi, S., Sheu, C. F., Zanetti, O., \& Frisoni, G. B. (2005). Distinctive clinical features of mild cognitive impairment with subcortical cerebrovascular disease. Dementia and Geriatric Cognitive Disorders, 19, 196-203.

Gardiner, J. M., Java, R. L., \& Richardson-Klavehn, A. (1998). Experiences of remembering, knowing, and guessing. Consciousness and Cognition, 7, 1-26.

Giovanello, K. S., \& Verfaellie, M. (2001). The relationship between recall and recognition in amnesia: Effects of matching recognition between patients with amnesia and controls. Neuropsychology, 15, 444-451.

Gomez-Isla, T., Price, J. L., McKeel, D. W., Morris, J. C., Growdon, J. H., \& Hyman, B. T. (1996). Profound loss of Layer II entorhinal cortex neurons distinguishes very mild Alzheimer's disease from nondemented aging. Journal of Neuroscience, 16, 4491-4500.

Grady, C. L., Haxby, J. V., Horwitz, B., Sundaram, M., Berg, G., Schapiro, M., et al. (1988). Longitudinal study of the early neuropsychological and cerebral metabolic changes in dementia of the Alzheimer type. Journal of Clinical and Experimental Neuropsychology, 10, 576-596.

Green, D. M., \& Swets, J. A. (1966). Signal detection theory and psychophysics. New York: Wiley.

Guillozet, A. L., Weintraub, S., Mash, D. C., \& Mesulam, M.-M. (2003). Neurofibrillary tangles, amyloid, and memory in aging and mild cognitive impairment. Archives of Neurology, 60, 729-736.

Henson, R. N. A., Casino, S., Herron, J. E., Robb, W. G. K., \& Rugg, M. D. (2003). A familiarity signal in human anterior medial temporal cortex. Hippocampus, 13, 301-304.

Henson, R. N. A., Rugg, M. D., Shallice, T., \& Dolan, R. J. (1999). Recollection and familiarity in recognition memory: An event-related functional magnetic resonance imaging study. Journal of Neuroscience, 19, 3962-3972.

Holdstock, J. S., Mayes, A. R., Roberts, N., Cezayirli, E., Isaac, C. L., O'Reilly, R. C., \& Norman, K. A. (2002). Under what conditions is recognition spared relative to recall after selective hippocampal damage in humans? Hippocampus, 12, 341-351.

Ibáñez, V., Pietrini, P., Alexander, G. E., Furey, M. L., Teichberg, D., Rajapakse, J. C., et al. (1998). Regional glucose metabolic abnormalities are not the result of atrophy in Alzheimer's disease. Neurology, 50, $1585-1593$.

Insausti, R., Juottonen, K., Soininen, H., Insausti, A. M., Partanen, K., Vainio, P., et al. (1998). MR volumetric analysis of the human entorhinal, perirhinal, and temporopolar cortices. American Journal of Neuroradiology, 4, 659-671.

Jorm, A. F. (1994). A short form of the Informant Questionnaire on Cognitive Decline in the Elderly (IQCODE): Development and crossvalidation. Psychological Medicine, 24, 145-153. 
Juottonen, K., Laakso, M. P., Insausti, R., Lehtovirta, M., Pitkanen, A., Partanen, K., \& Soininen, H. (1998). Volumes of the entorhinal and perirhinal cortices in Alzheimer's disease. Neurobiology of Aging, 19, $15-22$.

Kaplan, E., Goodglass, H. \& Weintraub, S. (1983). The Boston Naming Test. Philadelphia: Lea \& Febiger.

Khoe, W., Kroll, N. E. A., Yonelinas, A. P., Dobbins, I. G., \& Knight, R. T. (2000). The contribution of recollection and familiarity to yes-no and forced-choice recognition tests in healthy subjects and amnesics. Neuropsychologica, 38, 1333-1341.

Killiany, R. J., Moss, M. B., Albert, M. S., Sandor, T., Tieman, J., \& Jolesz, F. (1993). Temporal lobe regions on magnetic resonance imaging identify patients with early Alzheimer's disease. Archives of Neurology, 50, 949-954.

Knight, R. G. (1998). Controlled and automatic memory processes in Alzheimer's disease. Cortex, 34, 427-435.

Knowlton, B. J., \& Squire, L. R. (1995). Remembering and knowing: Two different expressions of declarative memory. Journal of Experimental Psychology: Learning, Memory, and Cognition, 21, 699-710.

Köhler, S., Black, S. E., Sinden, M., Szekely, C., Kidron, D., Parker, J. L., et al. (1998). Memory impairments associated with hippocampal versus parahippocampal-gyrus atrophy: An MR volumetry study in Alzheimer's disease. Neuropsychologia, 36, 901-914.

Koivisto, M., Portin, R., Seinela, A., \& Rinne, J. (1998). Automatic influences of memory in Alzheimer's disease. Cortex, 34, 209-219.

Kordower, J. H., Chu, Y., Stebbins, G. T., DeKosky, S. T., Cochran, E. J., Bennet, D., \& Mufson, E. J. (2001). Loss and atrophy of Layer II entorhinal cortex neurons in elderly people with mild cognitive impairment. Annals of Neurology, 49, 202-213.

Kroll, N. E. A., Yonelinas, A. P., Dobbins, I. G., \& Frederick, C. M. (2002). Separating sensitivity from response bias: Implications of comparisons of yes-no and forced-choice tests for models and measures of recognition memory. Journal of Experimental Psychology: General, $131,241-252$.

Leonard, B. W., Amaral, D. G., Squire, L. R., \& Zola-Morgan, S. (1995). Transient memory impairment in monkeys with bilateral lesions of the entorhinal cortex. Journal of Neuroscience, 15, 5637-5659.

Luis, C. A., Loewenstein, D. A., Acevedo, A., Barker, W. W., \& Duara, R. (2003). Mild cognitive impairment: Directions for future research. $\mathrm{Neu}$ rology, 61, 438-444.

Macmillan, N. A., \& Creelman, C. D. (1991). Detection theory: A user's guide. Cambridge, England: Cambridge University Press.

Mandler, G. (1980). Recognizing: The judgment of previous occurrence. Psychological Review, 87, 252-271.

Manns, J. R., Hopkins, R. O., Reed, J. M., Kitchener, E. G., \& Squire, L. R. (2003). Recognition memory and the human hippocampus. Neuron, 37, $171-180$.

Mayes, A. R., Holdstock, J. S., Isaac, C. L., Hunkin, N. M., \& Roberts, N. (2002). Relative sparing of item recognition memory in a patient with adult-onset damage limited to the hippocampus. Hippocampus, 12, 325340.

Mayes, A. R., Holdstock, J. S., Isaac, C. L., Montaldi, D., Grigor, J., Gummer, A., et al. (2004). Associative recognition in a patient with selective hippocampal lesions and relatively normal item recognition. Hippocampus, 14, 763-784.

McClelland, J. L., McNaughton, B. L., \& O'Reilly, R. C. (1995). Why there are complementary learning systems in the hippocampus and neocortex: Insights from the successes and failures of connectionist models of learning and memory. Psychological Review, 102, 419-457.

McKhann, G., Drachman, D., Folstein, M., Katzman, R., Price, D., \& Stadlan, E. M. (1984). Clinical diagnosis of Alzheimer's disease: Report of the NINCDS-ADRDA Work Group under the auspices of Department of Health and Human Services Task Force on Alzheimer's Disease. Neurology, 34, 939-944.
Mesulam, M.-M. (1999). Neuroplasticity failure in Alzheimer's disease: Bridging the gap between plaques and tangles. Neuron, 24, 521-529.

Mesulam, M.-M., Shaw, P., Mash, D., \& Weintraub, S. (2004). Taupathy in the cholinergic nucleus basalis is an early event in the progression from aging to MCI and AD. Annals of Neurology, 55, 815-828.

Morris, J. C. (1993). The Clinical Dementia Rating (CDR): Current version and scoring rules. Neurology, 43, 2412-2414.

Morris, J. C. (1996). The cognitive neuropsychology of Alzheimer-type dementia. New York: Oxford University Press.

Morris, J. C., Heyman, A., Mohs, R. C., Hughes, J. P., van Belle, G., Fillenbaum, G., et al. (1989). The Consortium to Establish a Registry for Alzheimer's Disease (CERAD): Pt. 1. Clinical and neuropsychological assessment of Alzheimer's disease. Neurology, 39, 1159-1165.

Norman, K. A., \& O'Reilly, R. C. (2003). Modeling hippocampal and neocortical contributions to recognition memory: A complementarylearning-systems approach. Psychological Review, 110, 611-646.

Pennanen, C., Kivipelto, M., Tuomainen, S., Hartikainen, P., Hänninen, T., Laakso, M., et al. (2004). Hippocampus and entorhinal cortex in mild cognitive impairment and early AD. Neurobiology of Aging, 25, 303310.

Petersen, R. C., Doody, R., Kurz, A., Mohs, R. C., Morris, J. C., Rabins, P. V., et al. (2001). Current concepts in mild cognitive impairment. Archives of Neurology, 58, 1985-1992.

Petersen, R. J. (2004), January/February). MCI as a useful clinical concept. Geriatric Times, 5(1), 15-18.

Price, J. L., \& Morris, J. C. (1999). Tangles and plaques in nondemented aging and "preclinical" Alzheimer's disease. Annals of Neurology, 45, $358-368$.

Ranganath, C., Yonelinas, A. P., Cohen, M. X., Dy, C. J., Tom, S. M., \& D'Esposito, M. (2004). Dissociable correlates of recollection and familiarity within the medial temporal lobes. Neuropsychologia, 42, 2-13.

Ratcliff, R., Sheu, C. F., \& Gronlund, S. (1992). Testing global memory models using ROC curves. Psychological Review, 99, 518-535.

Reitan, R. M. (1992). Trail Making Test: Manual for administration and scoring. Tucson, AZ: Reitan Neuropsychology Laboratory.

Reyna, V. F., \& Brainerd, C. J. (1995). Fuzzy-trace theory: An interim synthesis. Learning and Individual Differences, 7, 1-75.

Roman, G. C., Sachdev, P., Royall, D. R., Bullock, R. A., Orgogozo, J. M., Lopez-Pousa, S., et al. (2004). Vascular cognitive disorder: A new diagnostic category updating vascular cognitive impairment and vascular dementia. Journal of the Neurological Sciences, 226, 81-87.

Schacter, D. L., Norman, K. A., \& Koutstaal, W. (1998). The cognitive neuroscience of constructive memory. Annual Review of Psychology, 49, 289-318.

Seab, J. P., Jagust, W. J., Wong, S. T. S., Roos, M. S., Reed, B. R., \& Budinger, T. F. (1988). Quantitative NMR measurements of hippocampal atrophy in Alzheimer's disease. Magnetic Resonance in Medicine, 8, 200-208.

Snodgrass, J. G., \& Corwin, J. (1988). Pragmatics of measuring recognition memory: Applications to dementia and amnesia. Journal of Experimental Psychology: General, 177, 34-50.

Stoub, T. R., Bulgakova, M., Leurgans, S., Bennett, D. A., Fleishman, D., Turner, D. A., \& DeToledo-Morrell, L. (2005). MRI predictors of risk of incident Alzheimer disease: A longitudinal study. Neurology, 64, 15201524.

Tabert, M. H., Albert, S. M., Borukhova-Milov, L., Camacho, Y., Pelton, G., Liu, X., et al. (2002). Functional deficits in patients with mild cognitive impairment: Prediction of AD. Neurology, 58, 758-764.

Tendolkar, I., Schoenfeld, A., Golz, G., Fernández, G., Kuehl, K. P., Ferszt, \& H., Heinze, H.-J. (1999). Neural correlates of recognition memory with and without recollection in patients with Alzheimer's disease and healthy controls. Neuroscience Letters, 263, 45-48. 
Troncoso, J. C., Martin, L. J., Dal Forno, G., \& Kawas, C. H. (1996). Neuropathology in controls and demented subjects from the Baltimore Longitudinal Study of Aging. Neurobiology of Aging, 17, 365-371.

Tulving, E. (1985). Memory and consciousness. Canadian Psychologist, 26, 1-12.

Tulving, E. (1989). Remembering and knowing the past. American Scientist, 77, 361-367.

Vargha-Khadem, F., Gadian, D. G., Watkins, K. E., Connelly, A., Van Praesschen, W., \& Mishkin, M. (1997, July 18). Differential effects of early hippocampal pathology on episodic and semantic memory. Science, 277, 376-380.

Wechsler, D. (1987). The Wechsler Memory Scale—Revised manual. New York: Psychological Corporation.

West, M. J., Coleman, P. D., Flood, D. G., \& Troncoso, J. C. (1994). Differences in the pattern of hippocampal neuron loss in normal aging and Alzheimer's disease. Lancet, 444, 769-772.

Winblad, B., Palmer, K., Kivipelto, M., Jelic, V., Fratiglioni, L., Wahlund, L. O., et al. (2004). Mild cognitive impairment: Beyond controversies, towards a consensus-Report of the International Working Group on Mild Cognitive Impairment. Journal of Internal Medicine, 256, 240246.

Xu, Y., Jack, C. R., O’Brien, P. C., Kokmen, E., Smith, G. E., Ivnik, R., et al. (2000). Usefulness of MRI measures of entorhinal cortex versus hippocampus in AD. Neurology, 54, 1760-1767.

Yonelinas, A. P. (1994). Receiver-operating characteristics in recognition memory: Evidence for a dual-process model. Journal of Experimental Psychology: Learning, Memory, and Cognition, 20, 1341-1354.

Yonelinas, A. P. (1999). The contribution of recollection and familiarity to recognition and source-memory judgments: A formal dual-process model and an analysis of receiver operation characteristics. Journal of Experimental Psychology: Learning, Memory, and Cognition, 25, 14151434.

Yonelinas, A. P. (2002). The nature of recollection and familiarity: A review of 30 years of research. Journal of Memory and Language, 46, 441-517.

Yonelinas, A. P., Hopfinger, J. B., Buonocore, M. H., Kroll, N. E. A., \& Baynes, K. (2001). Hippocampal, parahippocampal, and occipital-temporal contributions to associative and item recognition memory: An fMRI study. NeuroReport, 12, 359-363.

Yonelinas, A. P., Kroll, N. E. A., Quamme, J. R., Lazzara, M. M., Sauvé, M. J., Widaman, K. F., \& Knight, R. T. (2002). Effects of extensive temporal lobe damage or mild hypoxia on recollection and familiarity. Nature Neuroscience, 5, 1236-1240.

Yovel, G., \& Paller, K. A. (2004). The neural basis of the butcher-on-thebus phenomenon: Familiarity and recollection in a face memory task. Neurolmage, 21, 789-800.

Zola-Morgan, S., Squire, L. R., Amaral, D. G., \& Suzuki, W. (1989). Lesions of perirhinal and parahippocampal cortex that spare amygdala and hippocampal formation produce severe memory impairment. Journal of Neuroscience, 9, 4355-4370.

Zola-Morgan, S., Squire, L. R., \& Ramus, S. (1994). Severity of memory impairment in monkeys as a function of locus and extent of damage within the medial temporal lobe memory system. Hippocampus, 4, 483-495.

Received July 13, 2005

Revision received September 26, 2005

Accepted October 17, 2005

\section{E-Mail Notification of Your Latest Issue Online!}

Would you like to know when the next issue of your favorite APA journal will be available online? This service is now available to you. Sign up at http://watson.apa.org/ notify/ and you will be notified by e-mail when issues of interest to you become available! 